\title{
La evaluación formativa y compartida durante el prácticum en la formación inicial delprofesorado: Análisis de un caso en Chile Formative and Shared Assessment during the practicum on the Pre-service Teacher Education: a case analysis in Chile

\author{
*Universidad de Los Lagos. Osorno (Chile), **Universidad Católica de Temuco y Universidad de Los Lagos. Osorno (Chile)
} \\ *Francisco Javier Gallardo-Fuentes, **Bastian Carter Thuillier
}

\begin{abstract}
Resumen. El objetivo de este artículo es analizar los resultados de la implementación de un sistema de evaluación formativa en el alumnado universitario chileno que cursa la carrera de pedagogía en educación física. Se lleva a cabo un diseño de estudio de casos con utilización de metodología mixta. Las técnicas de recogida de datos son: lectura y análisis de documentos; observación; conversación y narración. La muestra son 24 alumnos que cursan el prácticum y los correspondientes 24 profesores tutores de los centros educativos. Los principales resultados valoran la experiencia: (a) con una serie de ventajas (entre algunas que se valora como un sistema de evaluación poco injusto y los alumnos (as) señalan preferir las evaluaciones de procesos formativos) y inconvenientes de la experiencia, en donde se han obtenido resultados muy bajos, por lo que no se pueden considerar como tal; (b) se han encontrado diferencias estadísticamente significativas $(p=.02 *)$, entre la percepción del alumnado y los tutores en la variable de «trabajo de apoyo en el aula».

Palabras claves. Evaluación Formativa, Evaluación Compartida, Formación Inicial del Profesorado, Prácticum, Educación Física, Buenas Prácticas.
\end{abstract}

Abstract. The main purpose of this paper is to analyze the results of the implementation of a system about formative assessment on Physical Education Teacher Education student in Chile. A case study design with mixed methodology was designed. The data collection techniques are: reading and analysis of documents; observation; conversation and narration. The sample consists in 24 Students in the practicum and the corresponding 24 tutors in schools. The main results of the experience are: (a) with some advantages (some are valuated as an unfair method and Students show to prefer formative evaluation) and inconvenient of experience where very low results, then are not considered; (b) main differences are shown ( $p=.02^{*}$ ) between the students' perception in the variable of «support working on classes»

Keywords. Formative Assessment; Shared Assessment; Pre-service Teacher Education; Practicum; Physical Education; Best Practices.

\section{Introducción}

La educación está presente en cualquier estructura social moderna, y la sociedad chilena no es la excepción a esta realidad. Aunque de un tiempo a esta parte el protagonismo de la educación pasa por una etapa álgida, marcada por demandas y en donde actores de la formación primaria, secundaria y quienes se desempeñan en educación terciaria (Universitaria) se manifiestan exigiendo mejoras. Las universidades, como instituciones a cargo de la formación de nuevos profesionales de la educación, se encuentran enfocadas en dar respuesta a las demandas y ya asoman autores locales afirmando que:

La educación superior chilena actual se enfrenta a muchos desafíos propios de un mundo en continua transformación que impulsa a las universidades a mirarse críticamente en su interior para responder a las demandas de la sociedad de siglo XXI (Barrea, 2011: 33).

Las universidades chilenas han tenido que sustentar la labor declarada en sus correspondientes misiones institucionales. Así, dentro del país existen experiencias pioneras de innovación en la búsqueda de calidad y nuevos enfoques curriculares; casos como el de la Universidad de Talca, que en la búsqueda de calidad fue la primera en el país que diseña y adopta un enfoque formativo por competencias (Hawes \& Corvalán, 2005). Como consecuencia de la implementación de esta modalidad, las estructuras curriculares por competencias comienzan a extenderse y a instalarse en las instituciones de educación superior chilena, suponiendo un cambio de paradigma educativo, respondiendo a la tendencia actual y dar un vuelco hacia un paradigma centrado en el estudiante (Cabalín \& Navarro, 2008).

Dado el dinamismo que la educación terciaria adquiere es preciso profundizar en investigación y aumentar las vinculaciones con el medio, cooperación entre universidades, movilidad académica y estudiantil, incorporar la producción de innovaciones y conocimientos aplicados, así como la formulación de programas de formación y innovación curricular centrada en el aprendizaje del estudiante (Núñez, 2007).

Así, el profesor, en vez de imponer su criterio, se encuentra ante la necesidad de dialogar con los estudiantes, conocer sus intereses, ver

Fecha recepción: 25-07-15- Fecha envío revisores: 25-07-15- Fecha de aceptación: 18-12-15 Francisco Gallardo Fuentes

fgallardo@ulagos.cl qué aspectos les motivan, comprobar cuáles son sus preocupaciones, para luego integrar todo esto en la vida del aula (Barba, 2009:43)

No siempre estas innovaciones suponen por sí mismas el logro del éxito. Muchas veces las modificaciones curriculares y las innovaciones educativas responden a procesos de moda; por ejemplo, Díaz-Barriga y Lugo (2003) manifiestan que la innovación se podía interpretar de distintas maneras, pero la mayoría de las veces se da como resultado del incorporar las novedades educativas del momento, pero sin desarrollar trabajos de investigación y seguimiento de estas iniciativas en los espacios educativos. Es trascendental indagar a fondo y dar seguimiento a la acción del profesor, como actor principal que condiciona la construcción del aprendizaje del alumnado:

No en balde se espera que los profesores privilegien estrategias didácticas que conduzcan al alumnado a la adquisición de habilidades cognitivas de alto nivel, a la interiorización razonada de valores y actitudes, a la apropiación y puesta en práctica de aprendizajes complejos, resultado de su participación activa en ambientes educativos experienciales y situados en contextos reales (Díaz-Barriga, 2010: 51).

Por todo ello se ha llevado a cabo una búsqueda de experiencias que respondan a esta idea de complementar la innovación con la investigación en el aula, con la evaluación y seguimiento de las experiencias educativas innovadoras. Uno de los trabajos más sistemáticos en este sentido lo hemos encontrado en la llamada «Red de evaluación formativa y compartida en Educación Superior», formada en el año 2005 por un grupo de profesores universitarios (Hamodi, López \& López, 2014). Defienden que la evaluación formativa posee un gran potencial para insertarse en el proceso educativo de manera exitosa. Definen evaluación formativa como: «todo proceso de constatación, valoración y toma de decisiones cuya finalidad es optimizar el proceso de enseñanzaaprendizaje que tiene lugar, desde una perspectiva humanizadora y no como mero fin calificador». (López, 2009: 35).

Es así que la evaluación formativa se presenta como una herramienta adecuada para asegurar el éxito en el proceso enseñanza-aprendizaje (E-A), contando además con el potencial de vincular en el aula a todos los actores de este proceso E-A, si se utiliza como premisa fundamental la participación compartida entre el profesor y el alumno en lo que Flecha, Gómezy Puigvert (2001) llaman diálogo igualitario. Como hace referencia López (2009), la evaluación formativa es todo proceso que 
sirve para que el alumno aprenda más (y/o corrija sus errores) y para que el profesorado aprenda a trabajar mejor (a perfeccionar su práctica docente). Según este autor (López, 2009) la evaluación formativa y compartida tiene dinámicas dialógicas y evaluativas que establecemos con el alumnado sobre sus procesos de aprendizaje, en donde supone una comunicación tanto individualizada como grupal y que puede darse tanto en grupos pequeños como numerosos, lo que ya desde su conceptualización posee la condición de permitir que ambas partes profesoralumno cierren este proceso vinculante que es el proceso E-A. Estos autores proponen cinco líneas de acción para utilizar en el aula universitaria sistemas de evaluación formativa y compartida.

El cuaderno del profesor, actividades de practica especificas (como las practicas de campo), las producciones del alumnado y las fichas de seguimiento del alumnado, los procesos de autoevaluación (evaluación entre iguales y evaluación compartida) y dinámicas y ciclos de investigación y/o evaluación (López, 2012: 125)

Sin embargo, la evaluación formativa tiene un elemento básico que debe estar presente siempre: el feedback (Barrientos \& López, 2015). Hay un estudio que relaciona estrechamente el feedback con la evaluación formativa (Higgins, Hartley \& Skelton, 2002) en el encontraron que los estudiantes otorgaban mucha importancia al feedback que recibían, incluso cuando este provenía de interacciones tan simples como conocer las notas o respuestas correctas obtenidas en un examen; pero también cuando se trataba de entender algo de manera más profunda. Por su parte, Yorke (2005) afirma que las investigaciones muestran como la evaluación formativa puede crear un potente efecto en el aprendizaje de los estudiantes. Pintor, Martínez y Peire (2010) analizan una recopilación de buenas prácticas de evaluación formativa en educación superior, con una muestra de 2491 alumnos de 14 universidades españolas; los resultados encuentran algunas ventajas: los estudiantes tienen más motivación, se implican más, son más responsables de sus aprendizajes, muestran una mayor autonomía y valoran positivamente su participación en la evaluación. Por último, el estudio de Webber y Tschepikow (2011) demuestra también las ventajas de la evaluación formativa a la hora de promover el aprendizaje del alumnado, tras analizar datos recolectados durante 11 años.

En estudios que abordan innovaciones en torno a la forma de evaluar en educación nos encontramos con un concepto que se está usando para definir una intervención positiva, este concepto es el de «practica de éxito». Experiencias indican que la incorporación de metodologías participativas están siendo utilizadas en las intervenciones educativas ya que

Se produce en diálogos que son igualitarios, en interacciones en las que se reconoce la inteligencia cultural en todas las personas y que están orientadas a la transformación de los niveles previos de conocimiento y del contexto sociocultural para avanzar hacia el éxito de todos y todas (Aubert, Flecha, García, Flecha \& Racionero, 2009:167)

Así también se deja ver en el estudio de Herranz (2015) quien tras el uso sistemático autoevaluación, evaluación compartida, evaluación por pares y otorgando un papel activo y participativo a los alumnos logro documentar como su intervención se constituye como una práctica de éxito por las ventajas que esta interacción genera.

A partir de esta revisión del estado de la cuestión, hemos optado por aplicar procesos de evaluación formativa y compartida en el contexto educativo universitario chileno, concretamente en la Universidad de los Lagos, dentro de la asignatura del prácticum.

Por tanto, el objetivo de este artículo es presentar un ejemplo de «buena práctica» de evaluación formativa, y las ventajas, inconvenientes y diferencias obtenidas entre el profesorado y el alumnado, durante el periodo de prácticum en la formación inicial del profesorado de educación física en Chile.

\section{Metodología}

La experiencia de evaluación formativa en Formación Inicial del Profesorado

\section{Contexto}

El grupo de estudiantes que formó parte de la experiencia lo conformaban 24 alumnos que se encontraban cursando en el cuarto año de la carrera de Pedagogía en Educación Física de la universidad de los Lagos, ubicada en el sur de Chile, los 24 profesores tutores correspondientes a cada centro educativo en el que se realizaba el prácticum y los nueve docentes supervisores de la universidad.

Durante el prácticum el alumnado debía asistir por separado a diferentes centros educativos de la ciudad para trabajar con alumnos (as) de enseñanza media (equivaldría en España a la educación secundaria obligatoria (ESO) y bachillerato) junto a los respectivos profesores de Educación Física que existían en cada uno de los lugares. Cabe señalar que los profesores supervisores de la universidad y los docentes de cada centro sostenían al menos una reunión previa antes de que el estudiante iniciara su proceso de práctica.

Estos centros educativos sirven para la realización del prácticum pedagógico al alumnado universitario de FIP. Los establecimientos atienden a adolescentes de entre 14 años a 18 años y presentan diferentes formas al financiar su funcionamiento: (a) establecimientos de educación municipal (establecimientos educacionales financiados por el gobierno); (b) establecimientos de educación privada subvencionada (establecimientos educacionales financiados de manera compartida, tanto particular como financiados por el gobierno); (c) establecimientos de educación privada (financiados a través del cobro de mensualidades a su alumnado).

Sistema de evaluación formativa e instrumentos utilizados

El sistema de evaluación formativa que se utilizo estaba basado en lo propuesto por la red de evaluación formativa y compartida en docencia universitaria, procurando trabajar en a lo menos dos de las cinco líneas de acción propuestas por López (2012) y que son: el cuaderno del profesor, actividades de practica especificas (como las practicas de campo), las producciones del alumnado y las fichas de seguimiento del alumnado, los procesos de autoevaluación (evaluación entre iguales y evaluación compartida) y dinámicas y ciclos de investigación y/o evaluación.

El estudio responde a las múltiples experiencias que ubican a la utilización de procesos de evaluación formativa con resultados positivos, lo que encuentra semejanza con diferentes experiencias internacionales que se han presentado como evidencia de este tipo de procesos de innovación pedagógica (Biggs, 2005; Black \& Wiliam, 2006; Brown \& Pickford, 2013; Gibbs, 2003; Knight, 2006; Sambell \& Mcdowell, 2003).

El sistema de evaluación formativa utilizada en la intervención busca favorecer la interacción y feedback entre el alumnado, el profesor tutor del centro educativo y el profesor supervisor de la universidad, procurando favorecer el proceso formativo del alumnado. Para ello se procuro distribuir en un semestre que a su vez constituyo el espacio temporal de duración del prácticum una serie de acciones e instrumentos contenidos en la tabla 1 y que eran realizadas por el profesor de la universidad.

La experiencia se diseñó de tal manera que los alumnos recibieran todo el feedback posible durante su proceso formativo, con el apoyo de los instrumentos y las instancias que se detallan en la tabla 1. Para ello contaban, además, con un cuaderno de práctica que contenía los instrumentos antes señalados y en el cual debían llenar una ficha por cada sesión, con el respectivo plan y las reflexiones sobre lo ocurrido, con la posibilidad de ser retroalimentados por escrito constantemente, tanto por el profesor tutor del centro, como por el supervisor de la universidad.

Para dar el salto a la calificación se utilizo el «Cuestionario de valoración del prácticum», en donde el profesor tutor del centro valoraba el desempeño del alumnado en su proceso de prácticum y también el alumnado respondía a modo de autoevaluación. En base a los puntajes que cada alumno(a) obtenía al sumar el número likert obtenido en cada ítem, se asociaba esta a una escala de puntajes y notas calculada con un nivel de exigencia del $70 \%$ en una escala de calificación de cero a siete, que es la escala de calificación que se utiliza en Chile. El aprobado 
correspondía a un cuatro y los ciento veinte puntos correspondía a la nota máxima (siete), en un relación nota puntajes de $0=0$ puntos; $1=2$ puntos; $2=28$ puntos; $3=55$ puntos; $4=83$ puntos; $5=96$ puntos; $6=$ 108 puntos y $7=120$ puntos.

La calificación final se obtenía del promedio obtenido entre la calificación resultante del instrumento aplicado al profesor tutor y el instrumento que era aplicado al propio alumnado a modo de autoevaluación.

Durante el proceso de prácticum el alumnado era supervisado por un profesor de la universidad periódicamente (en adelante profesor supervisor), quien entregaba feedback en el mismo centro de práctica y sostenía una reunión con el profesor tutor del centro educativo, constituyéndose entre ambos y el alumnado un grupo de trabajo que debía encargarse de la formación práctica durante la estancia en el centro. Posterior a cada supervisión se realizaba una reunión entre el alumnado y el profesor supervisor de la universidad, esta reunión era grabada en formato audio y se centraba esencialmente en la entrega de retroalimentación por parte del docente universitario, así como la instancia de construir mutuamente con la participación activa del alumnado respuestas a situaciones pedagógicas emergentes relacionadas con el proceso práctico.

Finalmente el alumno diseñaba un informe referente a su propio prácticum, contrastando la información con autores de referencia, tomando como base la pauta que figura en la tabla 2. Este informe era presentado ante sus compañeros, utilizando una metodología participativa de análisis, que permitía la sistematización de la misma.

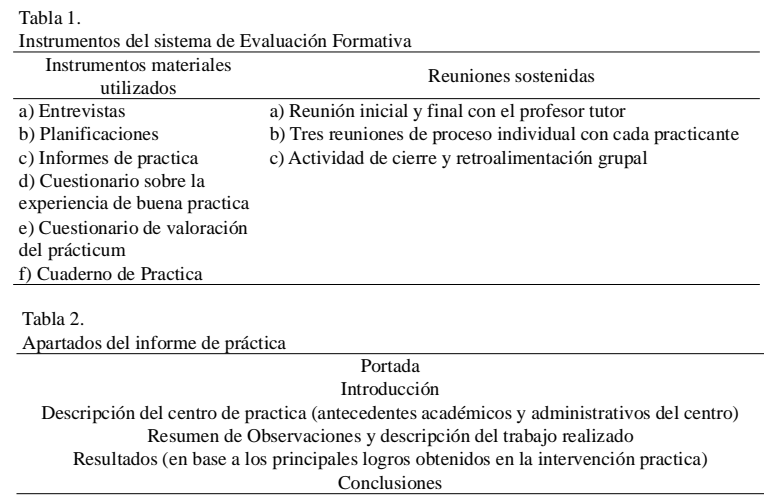

Recogiday análisis de datos para la evaluación de la experiencia

El diseño metodológico empleado en esta ocasión responde a un estudio de casos de tipo «intrínseco» entendiendo que la propia realidad estudiada en su particularidad representa ser un caso en sí mismo (Stake, 2010). Esta modalidad de estudio nos permite describir y analizar situaciones educativas específicas (Yin, 2009), desarrollando un trabajo intensivo y profundo de la situación a estudiar (Muñoz \& Muñoz, 2001). Esto último ofrece ventajas frente a otro tipo de metodologías al centrarse en las singularidades del contexto estudiado, permite desarrollar procesos investigativos a pequeña escala y en espacios de tiempo y recursos limitados (Latorre, Rincón \& Arnal, 1996).

Para efectuar el levantamiento de la información que permitiera determinar las ventajas, inconvenientes y diferencias entre el profesorado y el alumnado en la aplicación de un sistema de evaluación formativa y compartida en la titulación de Pedagogía en Educación Física de una universidad del sur de Chile, se utilizaron distintos instrumentos de recogida de datos que metodológicamente responderán al enfoque cualitativo.

Se aplicaron dos cuestionarios tipo likert y se llevaron a cabo entrevistas estructuradas. Con estos instrumentos se espera generar contrastes entre los resultados y obtener una mejor explicación del fenómeno durante la aplicación de este tipo de evaluaciones. Esta combinación de técnicas derivó en un enfoque que se adscribe a los métodos mixtos de investigación, dado este dialogo entre lo cualitativo y cuantitativo (Hernández, Fernández \& Baptista, 2010).

-Cuestionario sobre la experiencia de buena práctica. Fue aplicado con la finalidad de conocer las valoraciones que el alumnado le daba a la experiencia evaluativa de la que fueron parte. Era un cuestionario anónimoy se utilizo para la evaluación de la asignatura por parte del alumnado, está construido a partir del validado por Castejón, Pastor y Palacios (2015) con un CoeficienteAlpha de Cronbach de .84 para toda la escala. Para efectos de este estudio se utilizo el apartado que contenía las afirmaciones contenidas en la tabla 3. El apartado estaba constituido por 14 afirmaciones con respuestas en una escala de cinco grados, en donde $0=$ Nada; $1=$ Poco; 2= Algo; 3= Bastante; 4= Mucho y responde al grado de acuerdo del alumnado con las afirmaciones sobre evaluación formativa de la que tomaron parte.

- Cuestionario de valoración del prácticum. Su finalidad es recoger las valoraciones que otorgaba el profesor tutor del centro educativo al desempeño del alumno en proceso de prácticum, así como la autoevaluación del alumnado. Esto permite contrastar las dos valoraciones, y comprobar si existen diferencias medias entre alumnado y profesores tutores. Ambos contestaron de manera independiente y en la etapa final del proceso. El cuestionario contaba de 30 preguntas con respuestas en una escala tipo likert, de cuatro niveles, en donde 1= Nunca; 2= A veces; 3= Casi siempre y 4= Siempre. El cuestionario estaba compuesto por dos dimensiones principales: la dimensión personal y la dimensión profesional, que a su vez presentaban cinco y tres variables respectivamente, tal como se presenta en la tabla 4.

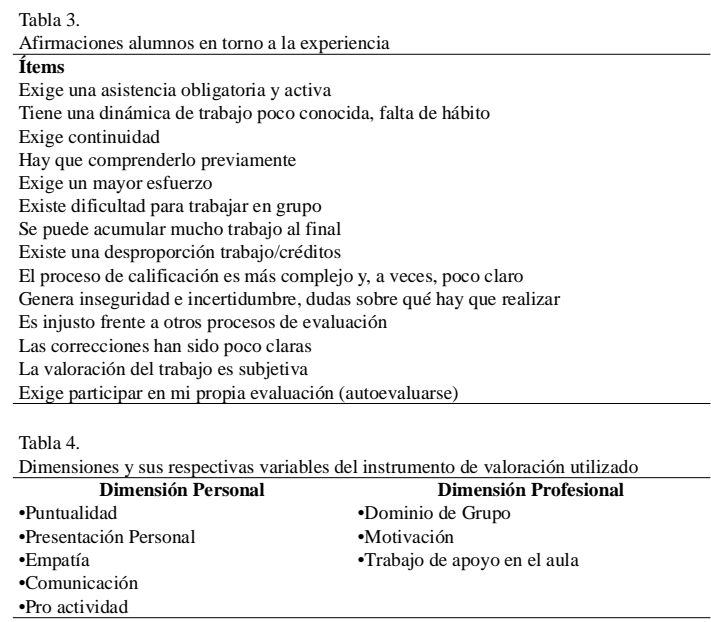

-Entrevistas estructuradas. Se basan en lo que nos dice Del Rincón, Arnal, Latorre y Sans (1995: 334) «la entrevista estructurada se refiere a una situación en la que un entrevistador pregunta a cada entrevistado una serie de interrogantes preestablecidos con una serie limitada de categorías de respuesta». La entrevista se aplico al alumnado en práctica así como a los profesores tutores del centro educativo y que participa-

Tabla 5

Entrevista aplicada al alumnado y al profesor tutor

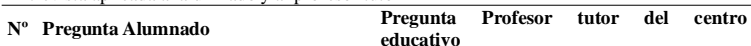

¿Qué es lo que más te ha gustado de la ¿Qué es lo que más te ha gustado de la

- experiencia de evaluación formativa en la experiencia de evaluación formativa en la que que has sido parte? has sido parte?

2. ¿Qué te ha aportado? ¿Qué crees le aporta al alumno en práctica y a ti como profesor Tutor?

Indica las actividades que más te han Indica las actividades que más te han gustado, 3. gustado, y también las que menos, y también las que menos, explicando por qué. explicando por qué. ¿Qué opinas sobre las reuniones con tu ¿Qué opinas sobre las reuniones que el alumno profesor supervisor al concretar una en práctica debe realizar con su profesor de la supervisión? ¿Has aprendido algo en esta universidad al concretar una supervisión? fase? ¿Qué crees que aprende en esta fase?

¿Qué opinas sobre las presentaciones ¿ ¿Qué opinas sobre que las presentaciones del ¿Qué opinas sobre las presentaciones del informe de practica el alumno deba realizarlo a informe a tus compañeros? ¿Has sus compañeros? ¿Qué crees que aprenderá en aprendido algo en esta fase?

6. ¿Qué te ha parecido el ser parte del ¿Qué te ha parecido el ser parte del proceso proceso evaluativo? evaluativo como profesor tutor? ¿Qué tipo de evaluaciones prefieres, las ¿Qué tipo de evaluaciones prefieres se le radicionales en donde vas solo a un realicen a los alumnos durante su prácticum,

7. examen final, o las que responden a las tradicionales en donde vas solo a un procesos formativos como el que has examen final, o las que responden a procesos $\begin{array}{ll}\text { respuesta. } & \text { Explica la razón de tu respuesta. } \\ \text { Crees que este tipo de evaluación aporta Crees que este tipo de evaluación aporta al }\end{array}$ al proceso formativo y futuro quehacer proceso formativo y futuro quob a 8. profesiont de an profesor? Explica la profesional de un prefesor? Explica la aź profesional de un profesor? Explica la profesional de un profesor? Explica la razó
razón de tu respuesta. 
ron del sistema de evaluación. Las preguntas giraban en torno a la experiencia de evaluación formativa.

Estas entrevistas buscaban construir la realidad a partir de los discursos y se conformaba de las preguntas contenidas en la tabla 5.

\section{Procedimiento}

La experiencia se llevo a cabo durante el segundo semestre del año académico 2014. En Chile el calendario académico anual escolar y universitario se desarrolla desde marzo a diciembre. Por tanto nuestro segundo semestre se lleva a cabo de agosto a diciembre. Los participantes fueron previamente informados de la investigación en la que iban a tomar parte, solicitando su permiso y dejando claro que al participar en la cumplimentación de los instrumentos (cuestionario y entrevista) debían ser contestados con sinceridad, teniendo la garantía de resguardo del anonimato en el tratamiento de los datos.

\section{Análisis de Datos}

En lo referido a la escala tipo likert, se utilizó estadística descriptiva por variable, obteniendo la desviación típica y media aritmética de las valoraciones del alumnado, este análisis de tipo cuantitativo fue utilizado en la presentación de los inconvenientes en torno a la experiencia. Los análisis se realizaron con el programa SPSS versión 18.0.

Para la presentación de las ventajas se realizo una explotación de datos cualitativos por medio del análisis de contenido, de manera tal que permita, visualizar los relatos en las categorías que fueron construidas de manera inicial, así como las que aparecieron de forma emergente a partir del proceso de codificación de la información y que están contenidas en la tabla 6.

Cada categoría será descrita y analizada en búsqueda de la presencia de feedback entre los actores de la experiencia basados en la tabla 7 que nos muestra el sistema de códigos. «Este tipo de análisis corresponde a una técnica de investigación destinada a formular, a partir de ciertos datos, inferencias reproducibles y válidas que puedan aplicarse a su contexto» (Krippendorf \& Wolfson, 1990: 28).

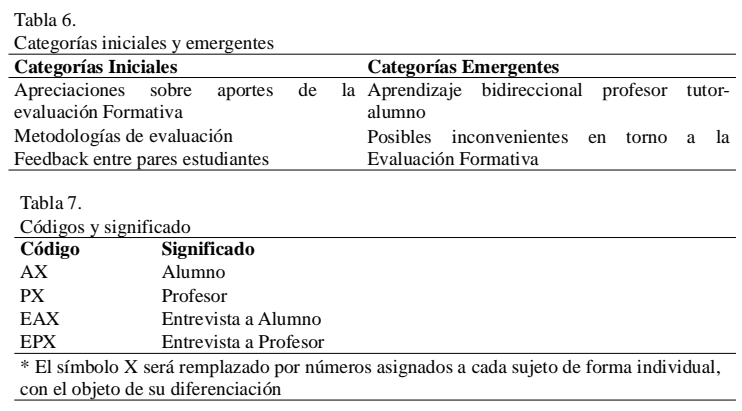

\section{Resultados y discusión}

Dentro de los resultados obtenidos podemos destacar que el grupo que tomo parte de la experiencia obtuvo un elevado rendimiento académico (6.36 puntos en una escala de 0-7; que equivale a un 9.08 en la escala de calificación española 0-10). También se obtuvo por medio de la entrevista un alto grado de satisfacción por parte del alumnado y de los profesores tutores.

Se analizó toda la información obtenida, considerando que en el proceso de recolección de datos se recogió información de carácter cuantitativo y cualitativo. Se han sistematizado los resultados de tal manera que se puedan establecer paralelos entre ambas fuentes de datos. Para lo anterior se ha segmentado la información en dos macroapartados: (a) ventajas e inconvenientes de la experiencia y (b) diferencias entre alumnos/tutores.

\section{Ventajas e inconvenientes de la experiencia}

Siete de las 14 afirmaciones contenidas en la tabla 8 se presentan bajo el valor dos, que es igual a algo; cuatro ítems entre algo y bastante y los otros tres ítems entre bastante y mucho, con lo que se encuentra mucha similitud con una experiencia que obtuvo un alto grado de acuer- do en una muestra 3013 estudiantes de diferentes universidades españolas (Romero-Martín, Castejón-Oliva \& López-Pastor, 2015).

En la tabla 8 podemos ver los datos referidos al grado de acuerdo con las afirmaciones que se pueden leer como posibles inconvenientes en torno a esta experiencia de tipo formativa.

El valor más próximo a bastante se dio en la Afirmación número cuatro (en adelante AF 4) hace referencia a que hay que comprender previamente el sistema evaluativo, a pesar de habernos cerciorado que se cumplieran los principios de transparencia y dominio durante todo el proceso, tal como hace referencia Álvarez-Méndez (2001).

En las afirmaciones que indagaban el grado de acuerdo sobre las exigencias que implica este sistema evaluativo nos encontramos que se ubicaron en su mayoría entre algo y bastante, ver tabla 8. Siendo las afirmaciones contenidas en los apartados (AF 1,3 y 14), las que obtuvieron mayores grados de acuerdo.

Tabla 8.

Afirmaciones y grado de acuerdo o desacuerdo del alumnado en torno al sistema de evaluación formativa.

1. Exige una asistencia obligatoria y activa

2. Tiene una dinámica de trabajo poco conocida, falta de hábito

3. Exige continuidad

4. Hay que comprenderlo previamente

5. Exige un mayor esfuerzo

6. Existe dificultad para trabajar en grupo

7. Se puede acumular mucho trabajo al final

8. Existe una desproporción trabajo/créditos

9. El proceso de calificación es más complejo y, a veces, poco claro

10. Genera inseguridad e incertidumbre, dudas sobre que hay que realizar

11. Es injusto frente a otros procesos de evaluación

11. Las correcciones han sido poco claras
13. La valo

trabajo es subjetiva

14. Exige participar en mi propia evaluación (autoevaluarse)

$\begin{array}{cc}\text { Media } & d t \\ 3.00 & .885 \\ 2.13 & 1.227 \\ 3.00 & .834 \\ 2.83 & .917 \\ 2.63 & .970 \\ 1.50 & 1.022 \\ 2.13 & .797 \\ 1.86 & .964 \\ 1.57 & .788 \\ 1.48 & .994 \\ 1.18 & .958 \\ 1.30 & 1.020 \\ 1.65 & 1.152 \\ 3.29 & .624\end{array}$

En torno al relato de los participantes del estudio, manifestaron en las entrevistas un alto grado de satisfacción con la experiencia: «Es una experiencia enriquecedora, ya que puedes compartir experiencias e ideas y enriquecer aun más nuestra práctica como docente» (EP, 10). Nos encontramos con este tipo de afirmaciones, con gran cantidad de referencias de parte del alumnado y profesores tutores sobre los aportes que la evaluación formativa entrega al proceso E-A, refuerzan más aun la importancia de que la evaluación no sea solo un proceso final, sino que debe estar dentro de todo el proceso E-A (Bordas \& Cabrera, 2001).

En otras afirmaciones se deja ver cómo este sistema de evaluación le permite al alumnado descubrir habilidades mediante la interacción práctica: «Me ha parecido muy bueno ya que soy yo quien ha vivido el proceso a cabalidad y con esto darme cuenta del potencial que poseo, así también de las debilidades que debo mejorar» (EA, 8).

En estudios previos se releva que sistemas de evaluación formativa generan estados de confianza y motivación del alumnado, así como una mayor implicación en su propio aprendizaje, dado su rol protagónico en el mismo proceso (Zaragoza, Luis-Pascual \& Manrique, 2009).

Por lo anterior podemos asumir que el feedback y la estructuración temporal es un elemento fundamental del sistema de evaluación, ya que permite corregir y retroalimentar en el proceso, en referencias en torno a la organización se menciona que: «Me ha aportado una mejor organización en cuanto al orden y estructuración de las clases. También el tener una mirada mucho más crítica y reflexiva de las mismas sesiones, sin duda favorece integralmente la formación docente» $(\mathrm{EA}, 16)$

Según nuestro análisis, estos resultados favorables hacia la experiencia obedecen al buen efecto que este tipo de experiencias tiene en el alumnado y que se contrasta con la excelente respuesta e idoneidad en contextos educativos, tal como se ha evidenciado en otras experiencias en donde se afirma que:

En los ciclos de investigación-acción desarrollados a lo largo de este tiempo, hemos comprobado en repetidas ocasiones que la propuesta es viable en un alto grado, y que es lo suficientemente flexible para que pueda ser adaptada a todo tipo de contexto y proceso educativo (López-Pastor, 2006: 40).

También se analizaron ocho afirmaciones presentadas en el «cuestionario sobre la experiencia de buena práctica» de manera negativa (AF 2, 6, 8, 9, 10,11,12 y 13). Bajo el criterio de que un alto grado de acuerdo a estas afirmaciones sería una muy mala señal hacia el sistema de evaluación formativa que se implementó. 
Los resultados dejaron ver una baja puntuación a las afirmaciones que fueron presentadas con esta intencionalidad (ver tabla 8).

Se puede constatar que incluso en el ítem 11 se presentó la valoración más baja (M=1.18); correspondiente al ítem que hace referencia a la posible injusticia frente a otros procesos de evaluación. De hecho los alumnos manifestaron estar entre nada o poco de acuerdo con estas afirmaciones. Sin embargo y como se pudo ver en la tabla 8, las afirmaciones que se refrían a la carga de trabajo (AF 5) y a las exigencias del sistema (AF 7) de evaluación formativa obtuvieron altas valoraciones, comportándose en contraste de manera similar a otros estudios que encontraron resistencia inicial en la aplicación de sistemas de evaluación formativa, principalmente por la carga de trabajo extra que esta supone (Zaragoza, Luis-Pascual \& Manrique, 2009) y en donde se resalta además que los sistemas de evaluación formativa sistemática pueden ser tediosos, sin embargo son promotores del ejercicio reflexivo del docente en torno a los aprendizajes del alumnado (Molinuevo, Sagardoy \& Gómez, 2009), aunque en la presente experiencia esto no interfirió en el alto grado de satisfacción que manifestaron los participantes de la experiencia.

A pesar de los inconvenientes que supuso la experiencia (mayoritariamente referidos a las exigencias del sistema de evaluación y a la carga de trabajo) el alumnado manifestó sentirse satisfecho con la misma, por lo que podemos entender que encontró más ventajas que dificultades.

En las entrevistas se sometió al alumnado a preguntas acerca de las preferencias evaluativas, poniendo a la evaluación formativa en contraste con las de corte tradicional. Los relatos surgidos ubican a la evaluación formativa en una posición de mejor consideración:

Prefiero las evaluaciones de procesos formativos. Ya que desde niños hemos sido formados con «exámenes finales»... cuesta salir de esa estructura. Pero claramente he aprendido mucho más de los procesos en los cuales debo reflexionar y obtener conocimientos de ello. (EA, 11).

En torno a lo anterior podemos encontrar antecedentes en una investigación que hace referencia a inconvenientes como las posibles resistencias por parte del alumnado, debido a la novedad que la evaluación formativa presenta (López-Pastor, 2006). En cambio, en los relatos del alumnado no aparecen referencias a este tipo de resistencias. De hecho, en los casos en que se encontraron relatos en torno a las evaluaciones de tipo tradicional, se utiliza la mención solo para contrastarla y relevar el valor de las de corte formativo: «Creo que las evaluaciones tradicionales son necesarias en determinados momentos, pero yo prefiero la formativa ya que acá el tiempo de evaluación se transforma en el tiempo de aprendizaje». (EA, 16).

\section{Diferencias en torno a las calificaciones entre alumno-tutores}

Como podemos ver en la tabla 9 el «Cuestionario de valoración del prácticum», arrojó que solo en una de las ocho variables se presentó una diferencia estadísticamente significativa entre profesores y el alumnado. Para la dimensión profesional se presentaron diferencias solo en la variable «trabajo de apoyo en el aula», con una significación del $p=.02 *$. Así los alumnos y profesores presentaron un comportamiento equivalente para siete de las ocho variables estudiadas, al contrario de lo que se encontró en el estudio de Tejeiro, Luis-Pascual \& Manrique (2012) en donde sí hubo diferencias entre la evaluación del profesor y la autoevaluación del alumnado.

Gran parte de la información recabada durante la experiencia pre-

Tabla 9

\begin{tabular}{cccccc}
\multicolumn{7}{c}{ U de Mann Whitney obtenidas entre X alumnos y X profesores. } \\
\hline & $\begin{array}{c}\mathrm{X} \\
\text { Profesores }\end{array}$ & $\begin{array}{c}\mathrm{X} \\
\text { Alumnos }\end{array}$ & $\begin{array}{c}\text { U de Mann } \\
\text { Whitney }\end{array}$ & $\mathrm{Z}$ & Significancia \\
\hline Dimensión personal & & & & & \\
Puntualidad & $3.9 \pm .3$ & $3.9 \pm .2$ & 278.5 & -.29 & .77 \\
Presentación Personal & $4.0 \pm .2$ & $4.0 \pm .1$ & 287.5 & -.03 & .98 \\
Empatía & $4.0 \pm .01$ & $4.0 \pm .1$ & 276.0 & -1.00 & .32 \\
Comunicación & $3.9 \pm .2$ & $3.9 \pm .2$ & 263.0 & -.73 & .47 \\
Pro actividad & $3.9 \pm 0.2$ & $3.9 \pm .1$ & 285.0 & -.07 & .94 \\
Dimensión Profesional & & & & & \\
Dominio de Grupo & $3.9 \pm .2$ & $3.9 \pm .2$ & 246.0 & -1.14 & .25 \\
Motivación & $3.9 \pm .2$ & $4.0 \pm .1$ & 261.0 & -.86 & .39 \\
Trabajo de apoyo en el aula & $4.0 \pm .1$ & $3.9 \pm .3$ & 215.5 & -2.31 &. $\mathbf{0 2 *}$
\end{tabular}

sentó un comportamiento común en torno a las valoraciones, así como a los relatos entre los profesores tutores y el alumnado, aunque hay autores como Lew, Alwis y Schmidt (2010: 147) quienes manifiestan que «por lo general, los estudiantes muestran una ejecución bastante mala a la hora de juzgar de forma adecuada su propio aprendizaje».

Solo la valoración del profesor tutor del centro educativo hacia el estudiante que desarrollo su prácticum y la auto-valoración del propio alumnado en proceso de prácticum dejó ver esta diferencia antes mencionada en una de las sub dimensiones, como se puede ver en la figura 1.

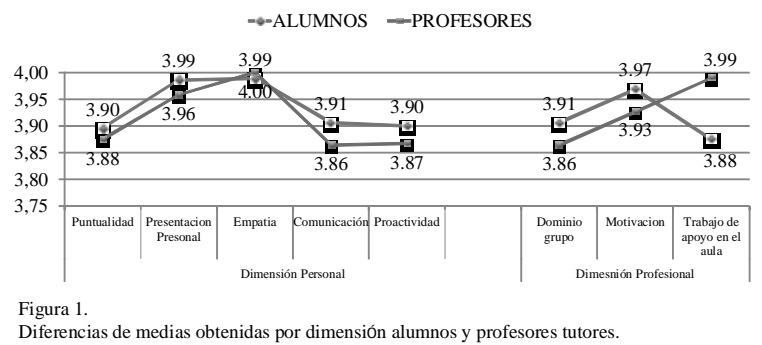

Los resultados de las medias obtenidas en la aplicación del «Cuestionario de valoración del prácticum», nos demuestran una muy buena disposición en cada una de las variables consideradas, tanto las dimensiones personal como profesional son evaluadas entre los 3.86 y los 3.99 puntos, acercándose de manera estrecha entre el alumnado y los profesores tutores del centro educativo.

En la figura 1 se observa que en la dimensión personal el alumnado ha obtenido una auto-valoración superior a la de sus tutores. Si bien los profesores tutores tienen una valoración más baja sobre los desempeños del alumnado durante sus procesos de prácticum, hay muy buenas valoraciones sobre el logro de los resultados propuestos. De la misma manera se comporta la dimensión profesional tomando en cuenta la diferencia presentada en la variable «Trabajo de apoyo en el aula», donde el alumnado, aunque lo considera muy bueno, está un poco por debajo de la valoración de sus tutores.

Desde el análisis cualitativo nos encontramos con relatos que podrían reforzar dichos resultados. Al analizar el relato de los profesores tutores surgen apreciaciones que sitúan la experiencia como un aporte, tanto desde el profesor tutor al alumnado, como también en sentido contrario, a manera de feedback bidireccional, valorando este vínculo profesor tutor- alumnado por:

El hecho de poder compartir ideas y experiencias a veces las metodologías que uno utiliza se pueden mezclar con las innovaciones que el alumno trae de la universidad, porque el feedback constante es lo que más me gustó. (EP, 23).

\section{Conclusiones}

Creemos que se ha cumplido en gran medida el objetivo planteado, que hacía referencia a presentar un ejemplo de «buena práctica» de evaluación formativa, y las ventajas e inconvenientes y diferencias obtenidas entre el profesorado y el alumnado, durante el periodo de prácticum en la formación inicial del profesorado de educación física en Chile, así lo muestran los resultados obtenidos. El alumnado encuentra que el sistema de evaluación posee más ventajas que desventajas, encontrándose solo valores que podrían catalogarse como inconvenientes en torno a la carga de trabajo y a las exigencias del sistema. En las entrevistas realizan variados comentarios positivos en torno a la experiencia, sin datos que nos permitieran detectar desventajas. Esto no coincide con otros estudios que sí han logrado detectar algunas desventajas.

Los datos parecen indicar una gran claridad en torno al sistema de evaluación formativa y se dio una interacción constante mediante el feedback. Esto se puede comprobar en la información recabada durante la experiencia y en el elevado grado de acuerdo en el instrumento aplicado a alumnos y profesores tutores. 
El trabajo podría ser importante para profesores que trabajan en docencia universitaria, especialmente en FIP; especialmente en la actual realidad educativa en que las universidades están transitando a modelos basados en competencias.

En prospectiva futura sería de gran ayuda la realización de estudios en torno a esta temática, por lo que se pretende incorporar este tipo de iniciativas, con el fin de construir nuevo conocimiento desde la base de la propia práctica educativa en otros módulos del itinerario formativo del estudiante de FIP de Educación Física en Chile. Respecto a esta línea además se pretende en futuros estudios recabar información en torno a la evaluación en la formación inicial del profesorado de educación física en chile, por medio de las percepciones de alumnos, egresados y profesores.

\section{Agradecimientos}

La presente experiencia no podría haber sido posible sin el respaldo de las unidades académicas de la Universidad de los Lagos, por lo que agradecemos al departamento de Ciencias de la Actividad física y a la Escuela de Pedagogía por su apoyo.

Pero sobre todo queremos agradecer al alumnado de FIP de educación física con año de egreso 2014 por su responsabilidad en el proceso. Así como a cada tutor de prácticum y a los centros educativos que participaron activamente del estudio.

\section{Referencias}

Álvarez-Méndez, J. M. (2001). Evaluar para conocer, examinar para excluir. Morata.

Aubert, A., Flecha, A., García, C., Flecha, R. \& Racionero, S. (2009). Aprendizaje dialógico en la Sociedad de la Información. Barcelona: Hipatia.

Barba, J. J. (2009). Redefiniendo la autoridad en el aula: Posibilidades para una educación democrática. Retos: nuevas tendencias en educación física, deporte y recreación, 15, 41-44.

Barrea, S. (2011): La reflexión docente como dinamizadora del cambio de prácticas en aula. Una experiencia de perfeccionamiento académico en la Universidad Católica Silva Henríquez (UCSH). Revista Perspectiva Educacional, 50, 31 60. Recuperado de http://www.redalyc.org/articulo.oa?id=333327289003.

Barrientos, E. \& López- Pastor, V. M. (2015) La evaluación formativa en educación superior. Una revisión internacional. Revista arbitrada del CIEG. 21, 272-284. Recuperado de http://www.grupocieg.org/archivos_revista/Ed.\%2021\%20(272284 ) \% 20 B arrientos \% 20 y \% 20 L \% C 3 \% B 3 pe z \% 20 \%20septiembre\%202015_articulo_id214.pdf

Biggs, J. (2005). Calidad del aprendizaje universitario. Madrid: Narcea.

Black, P. \& Wiliam, D. (2006). Assessment for Learning in the Classroom. En Gardner, J. (Ed.), Assessment and Learning (pp. 9-25). Londres: SAGE Publications.

Bordas, M. I. \& Cabrera, F. (2001). Estrategias de evaluación de los aprendizajes centrados en el proceso. Revista española de pedagogía, 218(25-48). Recuperado de http://cmapspublic3.ihmc.us/rid=1GLSW84JS-WYZWX0-H40/

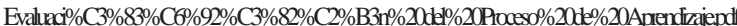

Brown, S. \& Pickforf, R. (2013). Evaluación de habilidades y competencias en Educación Superior. Madrid: Narcea.

Castejón-Oliva, F. J., Pastor, M. L. \& Palacios, A. (2015). Cuestionario sobre metodología y evaluación en formación inicial en educación física. Revista Internacional de Medicina y Ciencias de la Actividad Física y del Deporte, 15 (58), 245-267. Recuperado de http://fj7gg9gb2q.search.serialssolutions.com/ ?\&rft_id=info:doi/10.15366\%2Frimcafd2015.58.004

Cabalín Silva, D. \& Navarro Hernández, N. (2008). Conceptualización de los Estudiantes sobre el Buen Profesor Universitario en las Carreras de la Salud de la Universidad de La Frontera - Chile. International Journal of Morphology, 26, 887-892. Recuperado de http://dx.doi.org/10.4067/S071795022008000400017

Del Rincón, D., Arnal, J., Latorre, A. \& Sans, A. (1995). Técnicas de Investigación en Ciencias Sociales. Madrid: Dykinson.

Díaz-Barriga, F. (2010). Los profesores ante las innovaciones curriculares, en Revista Iberoamericana de Educación Superior (RIES), 1 (1), 37-57. Recuperado de https://ries.universia.net/article/viewFile/32/91.

Díaz-Barriga, F. \& Lugo, E. (2003). Desarrollo del currículo. En A. Diaz (Ed.), La investigación curricular en México: La década de los noventas (63-123). Ciudad de México: COMIE

Flecha, R., Gómez, J. \& Puigvert, L. (2001). Teoría Sociológica Contemporánea. Barcelona: Paidós.

Gibbs, G. (2003). Uso estratégico de la educación en el aprendizaje. En S. Brown y
A. Glasner (Eds), Evaluar en la universidad: problemas ynuevos enfoques (pp. 61-74). Narcea.

Hamodi, C., López, A. \& López-Pastor, V. M. (2014). Red de evaluación formativa y compartida en docencia universitaria: creación, consolidación y líneas de trabajo. REVALUE, 3(1). Recuperado de http://ojs.inee.edu.mx/revista/ index.php/revalue/article/view/110

Hawes, G. \& Corvalán, O. (2005). Construcción de un perfil profesional. Talca, Chile: Universidad de Talca. Recuperado de http://vrdp.utalca.cl/docs/pdf/ Construccion_Perfil_Profesional.pdf

Hernández, R., Fernández, C. \& Baptista, P. (2010). Metodología de la investigación ( $5^{\mathrm{a}}$ ed.). México: McGraw-Hill.

Herranz, M. (2015). Autoevaluación y evaluación compartida en el área de educación física. Aprendizaje y rendimiento académico en la etapa de educación primaria. Revista arbitrada del CIEG, 21, 126-141. Recuperado de http:// www.grupocieg.org/archivos_revista/Ed.\%2021\%20(126-141)\%20Marcos\% 20 Herranz\%20Sancho\%20\%20septiembre\%202015_articulo_id204.pdf

Higgins, R., Hartley, P. \& Skelton, A. (2002). The conscientious consumer: reconsidering the role of assessment feedback in student learning. Studies in Higher Education, 27(1), 53- 64.

Knight, P. T. (2006). El profesorado de educación superior: formación para la excelencia. Madrid: Narcea Ediciones.

Krippendorff, K. \& Wolfson, L. (1990). Metodología de análisis de contenido: teoría y práctica. Barcelona: Paidós.

Latorre, A., Del Rincón, D. \& Arnal, J. (1996). Bases metodológicas de la investigación educativa. Barcelona, España: Ediciones experiencia.

Lew, M. D., Alwis, W. A. M. \& Schmidt, H. G. (2010). Accuracy of students' self assessment and their beliefs about its utility. Assessment \& Evaluation in Higher Education, 35(2), 135-156.

López-Pastor, V. M. (2012). Evaluación formativa y compartida en la universidad: clarificación de conceptos y propuestas de intervención desde la Red Interuniversitaria de Evaluación Formativa. Psychology, Society, \& Education, 4, 117-130. Recuperado de http://www.psye.org/articulos/ vista\%20Lopez\%20Pastor.pdf.

López-Pastor, V. M. (coord..) (2009). Evaluación formativa y compartida en Educación Superior. Madrid España, Narcea, S.A. de ediciones.

López-Pastor, V. M., Aguado, R. M., García, J. G., López, E. M., Pastor, J. F,, Badiola, J. G. \& García, L. M. (2006). La Evaluación en Educación Física. Revisión de los modelos tradicionales y planteamiento de una alternativa: la evaluación formativa y compartida. Retos. Nuevas tendencias en Educación Física, Deporte y Recreación, 10, 31-41.

Molinuevo, J. S., Sagardoy, J. \& Gómez, M. J. (2009). Propuesta de evaluación del alumnado en educación física: un enfoque desde la Praxiología Motriz. Retos. Nuevas tendencias en educación física, deporte y recreación, 16, 41-45.

Muñoz, P. \& Muñoz, I. (2001). Intervención en la familia: estudio de casos. En G. Pérez(Ed.). Modelos de investigación cualitativa en educación social y animación sociocultural (pp.221-254). Madrid: Narcea.

Núñez, A. (2007). Escenario de la Educación Superior en Chile, visión actual y futuro. Algunas consideraciones. Santiago de Chile: Ministerio de Educación.

Pintor, P., Martínez, L. \& Peire, T. (2010). Sistemas y procedimientos de evaluación formativa en docencia universitaria: resultados de 34 casos aplicados durante el curso académico 2007-2008. ESE. Estudios Sobre Educación, 18, $255-276$.

Romero-Martín, R., Castejón-Oliva, F. J. \& López-Pastor, V. M. (2015). Divergencias del alumnado y del profesorado universitario sobre las dificultades para aplicar la evaluación formativa. RELIEVE-Revista Electrónica de Investigación y Evaluación Educativa, 21(1). Recuperado de http://www.uv.es/RELIEVE/ v21n1/RELIEVE v21n1_ME5.pdf.

Sambell, K. \& McDowell, L. (2003) La experiencia en la evaluación innovadora. En S. Brown y A. Glasner (Eds.), Evaluar en la Universidad (91-103). Madrid: Narcea.

Stake, R. E. (2010). Qualitative research: Studying how things work. Guilford Press.

Tejeiro, R. A., Gómez-Vallecillo, J. L., Romero, A. F., Pelegrina, M., Wallace, A. \& Emberley, E. (2012). La autoevaluación sumativa en la enseñanza superior: implicaciones de su inclusión en la nota final. Electronic Journal of Research in Educational Psychology, 10(27), 789-812.

Webber, K. L. \& Tschepikow, K. (2011). Learner-Centered Assessment: AComparison of Faculty Practices in US Colleges and Universities 1993 to 2004. Association for Institutional Research (NJ1). Recuperado de http://files.eric.ed.gov/fulltext/ ED531715.pdf.

Yin, R. (2009). Case Study Research. London: Sage.

Yorke, M. (2005). Formative Assessment in higher education: Its significance for employability, and steps towards its enhancement. Tertiary Education and Management, 11(3), 219-238. Recuperado de http://link.springer.com/article/ 10.1007\%2Fs11233-005-5110-z.

Zaragoza, J., Luis-Pascual, J. \& Manrique, J. (2009). Experiencias de innovación en docencia universitaria: resultados de la aplicación de sistemas de evaluación formativa. REDU,7(4), 1-33. Recuperado de http://red-u.net/redu/index.php/ REDU/article/view/111 